\title{
Nutrición parenteral: importancia de la determinación de hierro como impureza
}

\section{Parenteral nutrition: importance of iron as impurity}

\author{
Ana María Menéndez ${ }^{1,2}$, Silvia Sara Farías ${ }^{3}$, Roberto Enrique Servant ${ }^{3}$, \\ Adriana Ruth Weisstaub ${ }^{4}$, María Luz Pita Martín de Portela ${ }^{2,4}$.
}

Recibido: 5 de Julio de 2018. Aceptado para publicación: 15 de Agosto de 2018 https://doi.org/10.35454/rncm.v1n2.042

\section{Resumen}

Introducción: las mezclas para nutrición parenteral (NP) son fórmulas farmacéuticas complejas. Está demostrado que los componentes individuales utilizados para su preparación contienen microminerales esenciales como impurezas, incluyendo hierro, que pueden producir efectos adversos en el paciente y afectar la estabilidad de la NP.

Objetivos: determinar el nivel de hierro en los componentes individuales, disponibles en Argentina, para preparar mezclas de NP. Calcular la cantidad de hierro presente en fórmulas habituales de NP, destinadas a niños prematuros, pediátricos y adultos, preparadas con dichos componentes individuales.

Métodos: se determinó hierro en 48 productos comerciales, correspondientes a 13 componentes individuales utilizados para preparar NP, mediante Espectroscopía de Emisión Atómica Plasma Inductivo de Argón.

Resultados: el hierro se detectó en todos los componentes individuales analizados, excepto en el agua estéril. Los resultados fueron (promedio $\pm \mathrm{DE})(\mu \mathrm{g} / \mathrm{mL})$ : dextrosa $50 \%: 1,12 \pm 0,03$; dextrosa $70 \%$ : 1,32 \pm 0,52; aminoácidos $10 \%$ : 0,25 \pm 0,11; lípidos: 4,58 $\pm 0,80$; cloruro de potasio: $0,11 \pm 0,03$; cloruro de sodio: $0,11 \pm 0,03$; sulfato de magnesio: $0,11 \pm 0,00$; glicerofosfato de sodio: $2,76 \pm 0,48$; fosfato de sodio: $4,51 \pm 0,13$; gluconato de calcio: $2,01 \pm 0,27$; sulfato de zinc: $0,12 \pm 0,04$.

Conclusiones: las mayores cantidades de hierro se detectaron en los lípidos, fosfato de sodio, gluconato de calcio y

1 Cátedra de Farmacia Hospitalaria y Clínica. Carrera de Farmacia. Universidad de Belgrano, Ciudad Autónoma de Buenos Aires (CABA), Argentina.

2 Instituto Argentino de Educación e Investigación en Nutrición (IADEIN), Ciudad Autónoma de Buenos Aires.

Correspondencia: Ana María Menéndez. aname09@gmail.com

\section{Summary}

Introduction: Parenteral nutrition (PN) mixtures are complex pharmaceutical formulas. It is demonstrated that the individual components used for its preparation contain, as contaminants, essential micro minerals, including iron (Fe), which can produce adverse effects in the patient and affects the parenteral nutrition stability.

Objectives: Determine the Fe levels in the individual components, available in Argentina, to prepare PN mixtures. Calculate the Fe amount in regular formulas of $\mathrm{PN}$, prepared with these individual components, for preterm, pediatric and adult.

Methods: Iron was determined in 48 commercial products, corresponding to 12 individual components used to prepare PN using Argon Inductively Coupled-Plasma- Optical Emission Spectrometry - ICP/ OES (Perkin Elmer 5100 DV).

Results: Iron was detected in all the individual components, except in sterile water. The results were (mean $\pm S D)(\mu \mathrm{g} / \mathrm{mL})$ : dextrose $50 \%$ : $1.12 \pm 0.03$; dextrose $70 \%$ : $1.32 \pm 0.52$; amino acids $10 \%$ : $0.25 \pm 0.11$; lipids $20 \%: 4.58 \pm 0.80$; Potassium chloride: $0.11 \pm 0.03$; Sodium chloride: $0.11 \pm 0.03$; Magnesium sulfate: $0.11 \pm 0.00$; Sodium glycerophosphate, $2.76 \pm 0.48$; Sodium phosphate $4.51 \pm 0.13$; Calcium gluconate: $2.01 \pm 0.27$; zinc sulfate: $0.12 \pm 0.04$.

Conclusions: The highest amounts of $\mathrm{Fe}$ in the components were in the lipids, Sodium phosphate, Calcium gluconate and Sodium glycerophosphate. The amount of Fe in the mixtures

3 Departamento de Química. Comisión Nacional de Energía Atómica. San Martín, Provincia de Buenos Aires.

4 Cátedra de Nutrición, Facultad de Farmacia y Bioquímica, Universidad de Buenos Aires, Ciudad Autónoma de Buenos Aires (CABA). Argentina. 
glicerofosfato de sodio. Las cantidades calculadas en mezclas habituales de NP serían ( $\mu$ g de hierro/día): 74 (neonato de 1,2 Kg); 353 (niño de $10 \mathrm{Kg}$ ) y 2332 (adulto $60 \mathrm{~kg}$ ). Los lípidos y la dextrosa aportaron el mayor porcentaje del hierro total de dichas mezclas. Existen Farmacopeas que fijan límites de Fe como impurezas para algunos productos inyectables, pero no para las mezclas de NP preparadas con dichos componentes. Es importante conocer el contenido de microminerales en los productos utilizados para preparar NP, para evitar las deficiencias y los excesos, que pueden comprometer la evolución del paciente y afectar la estabilidad de la mezcla de NP.

Palabras clave: nutrición parenteral, hierro, impurezas de elementos, contaminación, estabilidad, prematuros, niños, adultos for PN ( $\mu \mathrm{g}$ of $\mathrm{Fe} /$ day) was: 74 (neonate of $1.2 \mathrm{Kg}$ ); 353 (child of $10 \mathrm{Kg}$ ) and 2332 (adult $60 \mathrm{~kg}$ ). In these mixtures, lipids and dextrose provided the highest percentage of total Fe. There are Pharmacopoeias that set limits of Fe as impurities for some injectable products, but not for mixtures of PN prepared with these components. It would be advisable to determine the amount of iron contained in the products, to avoid both deficiencies and excess, which may compromise the evolution of the critical patient and affect the stability of PN mixture.

Keywords: Parenteral Nutrition; Iron; Elemental impurities; Contamination; Stability; Preterm infants; Children; Adults.

\section{INTRODUCCIÓN}

La nutrición parenteral (NP) es una terapéutica de efectividad bien documentada para el cuidado y tratamiento de los pacientes. Es considerada uno de los principales avances de la medicina del siglo pasado, que ha posibilitado la sobrevivida de muchos pacientes en estado crítico, fundamentalmente, prematuros y recién nacidos de bajo peso ${ }^{(1)}$.

Las mezclas para NP son formulaciones complejas preparadas por los farmacéuticos, según prescripción médica, en el hospital o en centros de mezclas priva$\operatorname{dos}^{(2-4)}$. Aportan los nutrientes necesarios a aquellos pacientes con incapacidad para recibir, por su estado fisiopatológico, la alimentación oral. Contienen cantidades variables de numerosos compuestos químicos de acuerdo con el requerimiento de cada paciente y, su administración endovenosa, exige cuidados especiales por parte del equipo de la salud, con respecto a la prescripción, elaboración, dosis de sus componentes, esterilidad, compatibilidad, estabilidad y administración de la mezcla final ${ }^{(1,5)}$. En su elaboración se utilizan soluciones o emulsiones individuales y, en algunos casos, productos multicomponentes de macro y micronutrientes provistos por la industria farmacéutica ${ }^{(5)}$.

Los componentes individuales utilizados en la elaboración de mezclas de Nutrición Parenteral, así como las bolsas comerciales prellenadas, elaboradas por la industria farmacéutica, en la mayoría de los países no contienen oligoelementos declarados en los rótulos, salvo aquellos que los aportan específicamente. Sin embargo, se ha demostrado que algunos de estos componentes contienen, como impurezas contaminantes, microminerales esenciales y tóxicos $(\mathrm{Zn}, \mathrm{Cu}, \mathrm{Cr}$, Se, $\mathrm{Al}$, etc.), incluyendo hierro $(\mathrm{Fe})^{(6-10)}$. Las cantidades de dichas impurezas pueden ser clínica y nutricionalmente importantes para el paciente $y$, aún si están por debajo de los límites fijados por las respectivas Farmacopeas, es necesario que sean cuantificadas ${ }^{(11,12)}$. El grado de contaminación y toxicidad potencial dependen de la naturaleza del mineral, su abundancia y disponibilidad en el medio, el estado molecular específico y las condiciones fisicoquímicas de la mezcla $(\mathrm{pH}$, potencial redox, presencia de aniones y cationes, etc. $)^{(13)}$. La presencia de minerales proviene de contaminación no prevista, muy difícil de evitar y controlar por parte de la industria durante el proceso de fabricación. Las cantidades presentes varían según el fabricante, envase, componente analizado, lote (aún del mismo fabricante), fecha de vencimiento, etc. ${ }^{(6,7,9)}$. El Fe no es considerado un contaminante de riesgo ${ }^{(11)}$, aunque algunas Farmacopeas, como la de Argentina, solo establecen límites de Fe para muy pocos de estos componentes inyectables (gluconato de calcio, cloruro de sodio) $^{(13)}$.

$\mathrm{El} \mathrm{Fe}$ es uno de los oligoelementos o microminerales esenciales en el humano. Participa en el metabolismo energético y oxidativo ${ }^{(14)}$ y es necesario para el normal funcionamiento de los mecanismos de defensa del organismo a nivel celular, humoral y secretorio. El Fe funcional comprende la mayor proporción del Fe total corporal, formando parte del Hem, presente en la hemoglobina circulante, mioglobina y enzimas hemínicas. Una mínima cantidad de Fe no hemínico interviene en diversos sistemas enzimáticos. El Fe de reserva está unido a proteínas (ferritina y hemosiderina) en hígado, bazo, médula ósea y sistema retículo endotelial, siendo movilizado cuando las demandas no son cubiertas por la ingesta. El Fe no se encuentra libre en plasma y circula unido a la transferrina, proteína sintetizada por el hígado ${ }^{(15)}$. 
La deficiencia de $\mathrm{Fe}$ conduce a la utilización progresiva de sus depósitos, cuya depleción afecta en distinto grado las funciones Fe-dependientes. La sintomatología clínica se caracteriza por astenia, anorexia, fatiga, palidez de las mucosas y deterioro del rendimiento físico. Cuando las reservas se agotan se acentúan los signos clínicos y se manifiesta anemia microcítica hipocrómica. De manera concomitante, hay aumento de la susceptibilidad a las infecciones y alteraciones de la respuesta inmune y de las funciones neurológicas ${ }^{(16)}$.

Por otra parte, el exceso de Fe aumenta los depósitos de ferritina, los niveles plasmáticos de $\mathrm{Fe}$ y la saturación de la transferrina, facilitando su utilización por los microorganismos, permitiéndoles un mayor crecimiento y un proceso más virulento. Estos hechos explican la razón por la cual la terapia con Fe no siempre revierte, en el sistema inmune, los efectos de su deficiencia $y$, en algunos casos, se presenta aumento de la incidencia y virulencia de ciertas infecciones ${ }^{(17,18)}$. Por este motivo la presencia de Fe contaminante puede incrementar el aporte del $\mathrm{Fe}$ administrado con fines terapéuticos, conduciendo a resultados clínicos aparentemente contradictorios.

Además, si bien el Fe es un micromineral esencial, se aconseja no agregarlo a las fórmulas de NP debido a su interacción con otros nutrientes y se recomienda, realizar estudios de compatibilidad y estabilidad de las mezclas $^{(19,20)}$. La A.S.P.E.N (American Society for Parenteral and Enteral Nutrition) advierte acerca de los riesgos del exceso del $\mathrm{Fe}$ en los pacientes pediátricos y neonatos ${ }^{(21)}$. Por lo tanto, es de suma importancia conocer el contenido real de $\mathrm{Fe}$ en los componentes individuales y en las mezclas preparadas para NP con el objeto de modificar los aportes en las prescripciones, en función de las necesidades del paciente y evitar tanto las deficiencias como los excesos. Los objetivos del presente trabajo fueron: 1) Determinar el nivel de Fe en los componentes individuales disponibles para preparar mezclas de NP; 2) Calcular la cantidad total potencial de Fe administrada en fórmulas habituales de NP, preparadas con dichos componentes individuales, destinadas a niños prematuros, pediátricos y adultos. 3) Comparar los requerimientos de $\mathrm{Fe}$ con las cantidades que recibirían los pacientes en las mezclas de NP, con el que proviene de las impurezas no declaradas en los productos.

\section{MATERIALES Y MÉTODOS}

Productos comerciales estudiados: se determinó Fe en 48 productos comerciales, de diferentes laboratorios farmacéuticos y diversos lotes, correspondientes a 13 componentes individuales disponibles en Argentina y utilizados como nutrientes para preparar NP (Tabla 1).

Tabla 1. Productos estudiados y número de muestras

\begin{tabular}{|l|c|}
\hline \multicolumn{1}{|c|}{ Productos estudiados } & $\begin{array}{c}\text { Número de } \\
\text { muestras }\end{array}$ \\
\hline Dextrosa $70 \%$ & 5 \\
\hline Dextrosa $50 \%$ & 3 \\
\hline Aminoácidos $10 \%$ & 3 \\
\hline Aminoácidos pediátricos 10\% & 3 \\
\hline Lípidos 20 \% MCT/LCT & 3 \\
\hline Cloruro de sodio $20 \%$ & 5 \\
\hline Cloruro de potasio,1 mEq/mL) & 4 \\
\hline Sulfato de magnesio $25 \%$ & 5 \\
\hline Sulfato de zinc,1 mg/mL & 2 \\
\hline Gluconato de calcio & 6 \\
\hline Fosfato de sodio & 2 \\
\hline Agua estéril & 4 \\
\hline Glicerofosfato de sodio & 3 \\
\hline Total de productos estudiados & 48 \\
\hline
\end{tabular}

Nombre de Laboratorios estudiados: Rivero, BBraun, Surar-Pharma, Norgreen, Larian-Veinfar, FADA, Drawer, Fresenius.

Metodología de laboratorio: las determinaciones fueron realizadas por triplicado, en la Gerencia de Química de la Comisión Nacional de Energía Atómica (CNEA), mediante Espectroscopía de Emisión Atómica - Plasma Inductivo de Argón. Se utilizó un equipo ICP-OES OPTIMA 5100 DV Perkin Elmer (EEUU), provisto de detector de estado sólido (Segmented Coupled Charge Device - SCCD), utilizable en un rango espectral de unos $250 \mathrm{~nm}$ (240- $403 \mathrm{~nm}$ ). Se utilizó para este estudio un nebulizador de flujo cruzado asociado a una cámara de expansión tipo Scott y un auto-muestreador tipo Perkin Elmer AS90 Plus (EEUU). El método de ensayo fue optimizado y validado. Los parámetros instrumentales fueron optimizados contemplando aquellas variables críticas que influyen en la señal analítica sobre la línea de Fe 238.204 nm. Los parámetros de validación incluyeron límite de detección 0,003 $\mu \mathrm{g} /$ $\mathrm{mL}$, precisión intermedia $5 \%$ y sesgo: $8 \%$. El sesgo fue calculado usando material de referencia certificado de matriz NIST (SRM 1643 e) (EEUU) y la precisión fue 
evaluada usando diluciones apropiadas de estándares CertiPUR (Merck, Alemania) multielementales.

Las muestras con materia orgánica fueron digeridas en un sistema a microondas de laboratorio (Milestone mls 120 MEGA, Italia), utilizando ácido nítrico concentrado, grado analítico; luego, se realizaron las diluciones adecuadas con agua ultrapura 18,2 MWcm, obtenida mediante un equipo Nano Pure (Barnstead, Boston, MA, USA).

Análisis estadístico: los datos descriptivos se expresaron como promedio \pm desvío estándar $(\mathrm{DE})$ y rangos.

Contenido total de Fe de las mezclas de NP: fue calculado con base en los resultados promedio del contenido de Fe de los componentes individuales analizados y considerando la composición de fórmulas de prescripción habitual, que recibirían pacientes prematuros, niños y adultos (Tabla 2).

El Fe no fue determinado en las soluciones de sulfato de cobre, sulfato de manganeso, cloruro de cromo, ácido selenioso y vitaminas.

\section{RESULTADOS}

Las concentraciones de $\mathrm{Fe}$, expresadas en $\mu \mathrm{g} / \mathrm{mL}$, se observan en la Tabla 3, para los componentes estudiados de gran volumen y de pequeño volumen.

Los componentes de gran volumen analizados, excepto el agua estéril, contenían cantidades de $\mathrm{Fe}$ en un rango de $0,25 \pm 0,11$ y $4,58 \pm 0,80 \mu \mathrm{g} / \mathrm{mL}$. Los componentes de pequeño volumen analizados, contenían cantidades en un rango de $0,11 \pm 0,01$ y 4,51 $\pm 0,13 \mu \mathrm{g} /$ $\mathrm{mL}$, sin estar declarados en los rótulos de los productos.

Como puede observarse en la Tablas 3 las mayores cantidades de $\mathrm{Fe}$ se encontraron en los lípidos, fosfato de sodio, gluconato de calcio y glicerofosfato de sodio.

Considerando el promedio de los resultados encontrados, el contenido de Fe de una NP preparada para un neonato sería de $74 \mu \mathrm{g}$, para un niño de $524 \mu \mathrm{g}$, y el de una NP para un adulto de $2333 \mu \mathrm{g}$ (Tabla 4).

\section{DISCUSIÓN}

El contenido total de Fe como contaminante en las NP habituales, considerando el promedio de los resultados analíticos encontrados, totaliza: $74 \mu \mathrm{g}$ para un neonato de $1,2 \mathrm{~kg}$ de peso, $524 \mu \mathrm{g}$ para un niño de $10 \mathrm{~kg}$ de peso y 2333 $\mu \mathrm{g}$ en la NP destinada a un varón adulto de $60 \mathrm{Kg}$. Esas cifras de $\mathrm{Fe}$ no son consideradas por el equipo de salud, por no estar declaradas en los rótulos de los componen-
Tabla 2. Composición promedio de las mezclas de Nutrición Parenteral

\begin{tabular}{|c|c|c|c|}
\hline \multirow{2}{*}{ Gutrientes } & Neonatos\# & Niños* & Adultos $* *$ \\
\hline & \multicolumn{3}{|c|}{ Cantidad } \\
\hline Dextrosa 50 \% (g) & $12-15$ & $70-85$ & 350 \\
\hline Aminoácidos (g) & $3-4$ & 18 & 60 \\
\hline Lípidos al 20 \% (g) & $2,5-3,0$ & $10-15$ & 60 \\
\hline $\begin{array}{l}\text { Potasio (Cloruro) } \\
(\mathrm{mEq})\end{array}$ & $1,5-2$ & 15 & 60 \\
\hline Sodio (Cloruro) (mEq) & 2,25 & $14-18$ & 60 \\
\hline $\begin{array}{l}\text { Magnesio (Sulfato) } \\
(\mathrm{mEq})\end{array}$ & 0,6 & $3-5$ & 7 \\
\hline $\begin{array}{l}\text { Fósforo (Fosfato de } \\
\text { sodio) (mEq) }\end{array}$ & - & - & $45-54$ \\
\hline $\begin{array}{l}\text { Calcio (Gluconato) } \\
\text { (mEq) }\end{array}$ & 1,92 & 4,6 & 4,6 \\
\hline $\begin{array}{l}\text { Glicerofosfato de } \\
\text { sodio (mmol) }\end{array}$ & $-1,5$ & $10-15$ & - \\
\hline Zinc (mg) (Sulfato) & 0,36 & $3-4$ & 5 \\
\hline Cobre (mg) (Sulfato) & 0,024 & 0,5 & 0,5 \\
\hline $\begin{array}{l}\text { Manganeso }(\mu \mathrm{g}) \\
\text { (Sulfato) }\end{array}$ & 1,2 & 10 & 60 \\
\hline Cromo $(\mu \mathrm{g})$ (Cloruro) & 1,2 & 2 & 5 \\
\hline $\begin{array}{l}\text { Selenio }(\mu \mathrm{g}) \text { (Ácido } \\
\text { selenioso) }\end{array}$ & 1,2 & 10 & 60 \\
\hline $\begin{array}{l}\text { Molibdeno }(\mu \mathrm{g}) \\
\text { (Molibdato de amonio) }\end{array}$ & - & - & 25 \\
\hline Vitaminas (mL) & 1,2 & 5 & 5 \\
\hline $\begin{array}{l}\text { Agua estéril csp } \\
\text { volumen final (mL) }\end{array}$ & $70-170$ & $\begin{array}{c}1000- \\
1200\end{array}$ & $1500-2500$ \\
\hline
\end{tabular}

\# Fórmula promedio calculada para un neonato de $1200 \mathrm{~g}$.

* Fórmula promedio calculada para un niño de $10 \mathrm{~kg}$.

** Fórmula promedio calculada para un adulto: si se utiliza un producto multitraza (contiene $\mathrm{Fe}, \mathrm{Zn}, \mathrm{Cu}, \mathrm{Cr}, \mathrm{Mo}, \mathrm{Mn}, \mathrm{Se}, \mathrm{F}, \mathrm{I}$ ) se agregan $10 \mathrm{~mL}$ a la mezcla.

tes individuales utilizados en la preparación de la NP. Sin embargo, el Fe contaminante de la NP es incorporado al organismo por vía intravenosa (IV) y esa cantidad debería tenerse en cuenta en relación con los requerimientos de $\mathrm{Fe}$ (Tabla 5) y al aporte de $\mathrm{Fe}^{(22,23)}$, para controlar la evolución clínica y bioquímica del paciente que recibe alimentación por esa única vía (Figura 1). 
Tabla 3. Valores promedio de hierro en los componentes y rangos (entre paréntesis)

\begin{tabular}{|c|c|}
\hline $\begin{array}{l}\text { Componente de gran volumen } \\
\text { (Volumen del envase) }\end{array}$ & $\begin{array}{l}\text { Hierro }(\mu \mathrm{g} / \mathrm{mL}) \\
\text { Promedio } \pm \mathrm{DE}\end{array}$ \\
\hline Dextrosa $50 \%$ (500 mL) & $1,26 \pm 0,32(0,705-1,718)$ \\
\hline Dextrosa $70 \%$ (500 mL) & $1,32 \pm 0,52(1,020-1,920)$ \\
\hline Aminoácidos $10 \%$ ( $500 \mathrm{~mL}$ ) & $0,25 \pm 0,11(0,075-0,450)$ \\
\hline Lípidos 20 \% (500 mL) & $4,58 \pm 0,80(3,53-5,72)$ \\
\hline Agua estéril (500 mL) & No detectable \\
\hline $\begin{array}{c}\text { Componente de pequeño } \\
\text { volumen } \\
\text { (Volumen del envase) }\end{array}$ & $\begin{array}{l}\text { Hierro }(\mu \mathrm{g} / \mathrm{mL}) \\
\text { Promedio } \pm \mathrm{DE}\end{array}$ \\
\hline $\begin{array}{l}\text { Potasio cloruro, } 3 \mathrm{mEq} / \mathrm{mL} \text { (amp. } \\
\times 5 \mathrm{~mL} \text { ) }\end{array}$ & $0,11 \pm 0,03(0,105-0,110)$ \\
\hline Sodio cloruro $20 \%$ (amp. x $10 \mathrm{~mL}$ ) & $0,11 \pm 0,03(0,105-0,110)$ \\
\hline $\begin{array}{l}\text { Magnesio sulfato } 25 \% \text { (amp. x } \\
5 \mathrm{~mL} \text { ) }\end{array}$ & $0,11 \pm 0,01(0,105-0,110)$ \\
\hline $\begin{array}{l}\text { Fosfato de sodio (Frasco amp. } x \\
10 \mathrm{~mL} \text { ) }\end{array}$ & $4,51 \pm 0,13(4,29-4,69)$ \\
\hline $\begin{array}{l}\text { Glicerofosfato de sodio (Frasco } \\
\text { amp. x } 20 \mathrm{~mL} \text { ) }\end{array}$ & $2,76 \pm 0,48(2,17-3,47)$ \\
\hline $\begin{array}{l}\text { Calcio gluconato } 20 \% \text { (amp. x } \\
10 \mathrm{~mL} \text { ) }\end{array}$ & $2,01 \pm 0,27(1,61-2,49)$ \\
\hline $\begin{array}{l}\text { Zinc sulfato, } 1 \mathrm{mg} / \mathrm{mL} \text { (amp. x } 10 \\
\mathrm{~mL} \text { ) }\end{array}$ & $0,12 \pm 0,04(0,120-0,125)$ \\
\hline
\end{tabular}

Para el análisis de los resultados del presente trabajo se han considerado las necesidades de Fe de un neonato, de un niño de $10 \mathrm{Kg}$ de peso y en el caso del adulto, se ha elegido un varón porque los requerimientos de la mujer dependen de las distintas etapas fisiológicas.

\section{Aporte de Fe en la NP y requerimientos del neonato}

El contenido de Fe presente en la NP de neonatos $(74 \mu \mathrm{g})$ es una cantidad muy baja, en relación con los requerimientos de los prematuros (Figura 1) para mantener el balance aconsejado de $\mathrm{Fe}(200 \mu \mathrm{g} / \mathrm{Kg} / \mathrm{d})$ ${ }^{(22)}$. Las pequeñas cantidades aportadas como impurezas podrían ser incorporadas al metabolismo, pero se requieren estudios sobre el tema. Los recién nacidos de muy bajo peso al nacer $(<1.500 \mathrm{~g})$, en general, reciben nutrición parenteral sin agregado de $\mathrm{Fe}$ intravenoso ${ }^{(24)}$.
Tabla 4. Hierro en los componentes individuales y Fe total en las mezclas de NP para neonatos, niños y adultos

\begin{tabular}{|c|c|c|c|}
\hline & Neonato & Niño & Adulto \\
\hline Producto & \multicolumn{3}{|c|}{ Hierro $(\mu \mathrm{g})$} \\
\hline Dextrosa $70 \%$ & 20,74 & -- & -- \\
\hline Dextrosa $50 \%$ & -- & 217,8 & 732 \\
\hline Aminoácidos $10 \%$ & 10,5 & 38,75 & 175 \\
\hline Lípidos $20 \%$ & 27,48 & 235,87 & 1374 \\
\hline Fosfato de sodio & -- & 4,64 & 22,7 \\
\hline Glicerofosfato de sodio & 4,2 & -- & -- \\
\hline Cloruro de sodio $20 \%$ & 0,04 & 1,00 & 1,88 \\
\hline Cloruro de potasio & 0,13 & 2,83 & 6,36 \\
\hline Sulfato de magnesio $25 \%$ & 0,03 & 0,22 & 0,36 \\
\hline Gluconato de calcio $20 \%$ & 10,51 & 22,56 & 20,1 \\
\hline Sulfato de zinc & 0,04 & 0,13 & 0,57 \\
\hline Agua estéril & ND & ND & ND \\
\hline Fe Total $(\mu \mathrm{g})$ & 74 & 524 & 2333 \\
\hline Volumen Total (mL) & 166 & 1000 & 2000 \\
\hline Fe $\mu \mathrm{g}$ por Litro de NP & 444 & 524 & 1176 \\
\hline
\end{tabular}

-- : no utilizado en la fórmula de NP

ND: no detectable

Los valores promedio de hemoglobina en los recién nacidos de muy bajo peso al nacer $(<1.000 \mathrm{~g})$ son aproximadamente $16,5 \mathrm{~g} / \mathrm{dL}$ y disminuyen durante los primeros 3 meses de vida ${ }^{(25-26)}$. Luego, existe una disminución progresiva de hemoglobina durante el primer mes de vida extrauterina, debido a que no se ha logrado depósito de Fe en el último trimestre del embarazo y las demandas son elevadas por la expansión de la masa metabólicamente activa que implica el crecimiento.

En el caso de una NP a largo plazo, el neonato necesita retener mayor cantidad de $\mathrm{Fe}$, según el peso de nacimiento y la velocidad de crecimiento, pero en esos casos se implementan estrategias tales como la administración de transfusiones de glóbulos rojos, hasta cuando pueda tolerar la nutrición enteral temprana con la administración de suplementos ${ }^{(27)}$. 
Fe en la NP y requerimientos de Fe en niños nacidos a término

Los requerimientos de $\mathrm{Fe}$ a partir del nacimiento a término representan las necesidades para el reemplazo de las pérdidas inevitables más las necesarias para el crecimiento (Tabla 5) ${ }^{(22,23)}$ y serían las cantidades para administrar por vía IV (Figura 1).

El recién nacido a término tiene altos niveles de hemoglobina y de Fe corporal. Sin embargo, durante los primeros 6 meses existe depleción de los depósitos de $\mathrm{Fe}$, como consecuencia de la elevada velocidad de ganancia de peso y de la baja concentración de Fe en la leche materna ${ }^{(27,28)}$.

La contaminación con Fe calculada para la NP de un niño de $10 \mathrm{Kg}$ de peso sería de $524 \mu \mathrm{g}$, que representa $55 \%$ de los requerimientos diarios $(960 \mu \mathrm{g} / \mathrm{d})$, durante el primer año de vida (Tabla 5). Por otra parte, el Fe de contaminación representa un aporte de $52,4 \mu \mathrm{g} / \mathrm{Kg}$ de peso del niño.

Tabla 5. Requerimientos de hierro. Cifras correspondientes al hierro que debe ser absorbido (22-23)

\begin{tabular}{|c|c|c|c|c|c|}
\hline \multirow[t]{2}{*}{ Grupo/Edad (años) } & \multirow{2}{*}{$\begin{array}{c}\text { Crecimiento } \\
\text { Mediana } \\
\mu \mathrm{g} / \text { día }\end{array}$} & \multicolumn{2}{|c|}{$\begin{array}{l}\text { Pérdidas } \\
\text { Mediana }\end{array}$} & \multicolumn{2}{|c|}{$\begin{array}{c}\text { Total } \\
\text { Percentilo } 95\end{array}$} \\
\hline & & $\begin{array}{c}\text { Obligatorias } \\
\mu \mathrm{g} / \text { día }\end{array}$ & $\begin{array}{c}\text { Menstruales } \\
\mu \mathrm{g} / \mathrm{d} \text { día }\end{array}$ & $\mu g / d i ́ a$ & $\mu \mathrm{g} /$ día \\
\hline \multicolumn{6}{|l|}{ Niños } \\
\hline $0.25-1$ & 560 & 210 & & 960 & 120 \\
\hline $1-2$ & 240 & 250 & & 610 & 56 \\
\hline \multicolumn{6}{|l|}{ Adultos } \\
\hline Varones & & 910 & & 1140 & 18 \\
\hline \multicolumn{6}{|l|}{ Mujeres } \\
\hline Edad fértil & & 770 & 1600 & 2370 & 24 \\
\hline Posmenopausia & & 770 & & 960 & 18 \\
\hline
\end{tabular}

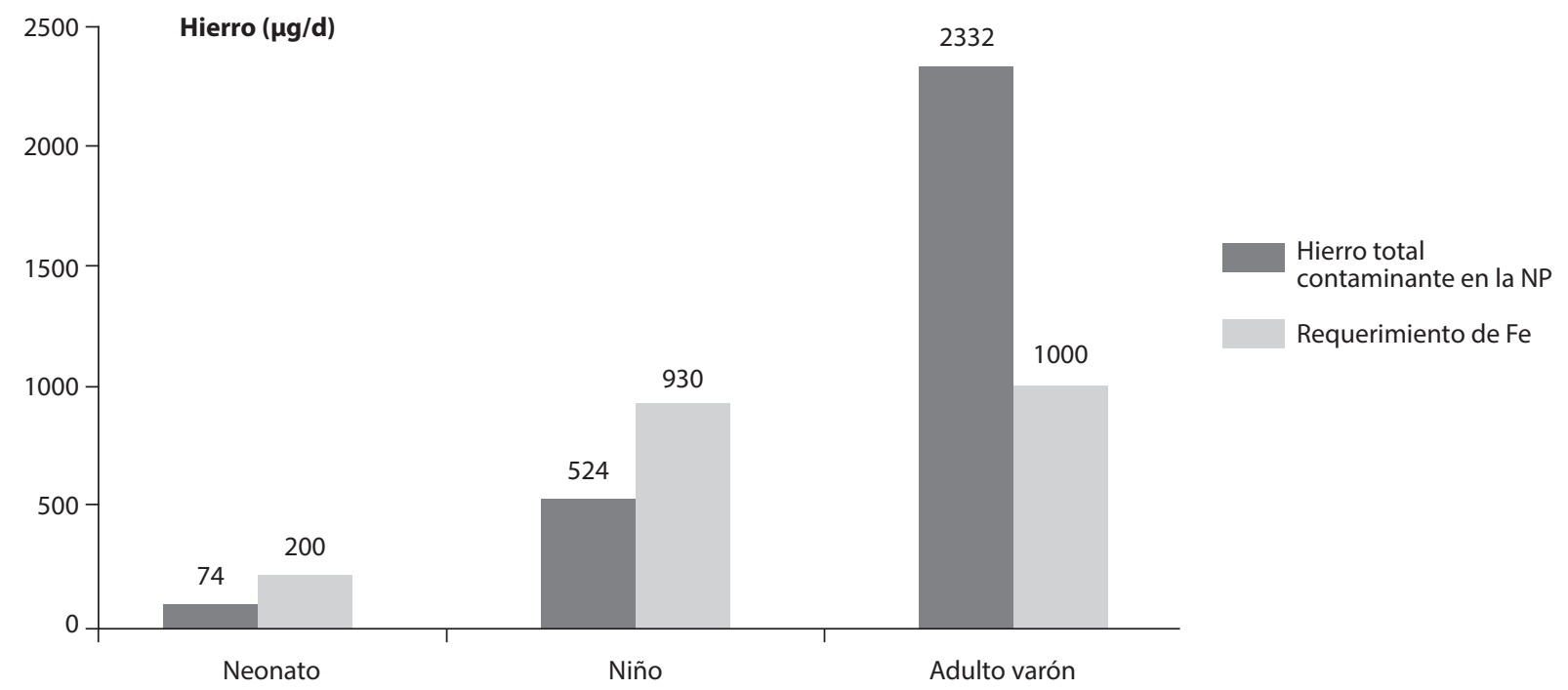

Figura 1. Contenido total de Fe como contaminante en las NP en relación a los requerimientos de $\mathrm{Fe}^{(22-23)}$ 


\section{Aporte de Fe en la NP y requerimientos en el adulto}

En la NP del adulto el aporte total de Fe que proviene de las impurezas $(2333 \mu \mathrm{g})$ es el doble de los requerimientos diarios del varón $(1140 \mu \mathrm{g} / \mathrm{d})($ Figura 1) y alcanzaría para cubrir las necesidades de una mujer en edad fértil (Tabla 5). En el caso del varón adulto y de la mujer posmenopáusica las elevadas cifras de contaminación con Fe podrían producir efectos adversos y comprometer la evolución del paciente. La sobrecarga de Fe por vía IV satura la capacidad de unión del Fe a la transferrina y produce efectos adversos en los pacientes. Este oligoelemento en exceso puede favorecer un mayor crecimiento de los microorganismos, generar un proceso más virulento y por lo tanto, disminuir la función inmunitaria ${ }^{(18)}$.

Cuando se agregan a la NP productos multitraza para adultos, que contienen, entre otros oligoelementos $2 \mathrm{mg}$ de $\mathrm{Fe}$, la cantidad total aportada sería de 4,2 $\mathrm{mg} /$ día. Esta cantidad será excesiva tanto en relación con los requerimientos como a la estabilidad de los lípidos y puede promover reacciones de oxidación de ciertos aminoácidos y vitaminas.

\section{EFECTOS ADVERSOS DEL HIERRO}

El Fe puede presentarse como ferroso o férrico $\left(\mathrm{Fe}^{2+} \leftrightarrow\right.$ $\mathrm{Fe}^{3+}$ ) lo cual le confiere la propiedad de ser un potente catalizador de reacciones que generan radicales libres en presencia de oxígeno molecular.

$$
\begin{gathered}
\mathrm{Fe}^{2+}+\mathrm{H}_{2} \mathrm{O}_{2} \rightarrow \mathrm{OH}^{-}+{ }^{*} \mathrm{OH}+\mathrm{Fe}^{3+} \\
\mathrm{Fe}^{3+}+\mathrm{AH} \rightarrow \mathrm{Fe}^{2+}+\mathrm{A}^{*}+\mathrm{H}^{+} \\
\mathrm{Fe}^{2+}+\mathrm{O}_{2} \rightarrow \mathrm{O}_{2}+\mathrm{Fe}^{3+}
\end{gathered}
$$

Estas reacciones inician la peroxidación en cadena sobre los dobles enlaces de los ácidos grasos poliinsaturados de las membranas celulares, produciendo alteraciones celulares ${ }^{(29)}$. También estas reacciones pueden producirse en las mezclas de NP, disminuyendo la vida útil de los productos y la biodisponibilidad de otros nutrientes ${ }^{(30)}$.

Cuando existe sobrecarga de Fe se excede la capacidad de almacenamiento de Fe, como ferritina, aumentando la saturación de la transferrina y el Fe circulante, lo que promueve la formación de radicales libres y el riesgo de peroxidación lipídica. Estas reacciones explican los efectos adversos de daño isquémico del miocardio en animales de laboratorio ${ }^{(31)}$, el aumento del riesgo de enfermedad coronaria en el hombre ${ }^{(32)}$ y la retinopatía asociada a prematurez ${ }^{(33,34)}$.

Por este motivo, la administración indiscriminada de hierro con fines terapéuticos ha conducido a resultados aparentemente contradictorios, cuyo esclarecimiento demanda un minucioso estudio experimental.

\section{ESTABILIDAD DE LA NP CON AGREGADO DE HIERRO}

En el presente trabajo, el mayor nivel de contaminación con $\mathrm{Fe}$, en los componentes analizados, se encontró en los lípidos, fosfato de sodio, gluconato de calcio y glicerofosfato de sodio (Tabla 3). Por otra parte, al considerar el Fe total de contaminación en las mezclas de NP, las soluciones de dextrosa y de lípidos contribuyeron con las más altas cantidades (Tabla 4) y el mayor porcentaje, por los volúmenes utilizados en relación a los otros componentes (Figura 2). Sin embargo, el Fe presente en el gluconato de calcio, fosfato de sodio y glicerofosfato de sodio debería ser cuantificado y contabilizado, aún si estuviese por debajo del límite considerado por la Farmacopea Argentina VII edición.

Las cifras de contaminación con $\mathrm{Fe}$ en las mezclas NP del presente trabajo representan, en relación con su volumen, entre 444 y $1176 \mu \mathrm{g}$ de Fe/L, según se considere la NP para un neonato o un adulto. Estos valores están en el rango de los publicados por otros autores $(0,025$ y $1370 \mu \mathrm{g} / \mathrm{L})$ referentes a la contaminación con Fe en mezclas de nutrición parenteral, dependiendo del fabricante y el número de lote de los componentes utilizados para la preparación ${ }^{(6,35)}$.

Como ya se mencionó, la propiedad del Fe de generar radicales libres es responsable de su capacidad de catalizar reacciones de oxidación de aminoácidos y lípidos. Los lípidos peroxidados inician una serie de reacciones químicas que provocan oxidación de las vitaminas liposolubles y a la vez, pérdidas de ácidos grasos esenciales $^{(19,29,36)}$. En consecuencia, en las mezclas de NP se produce desestabilización de los lípidos dando lugar a un aumento del tamaño de los glóbulos que pueden ser perjudiciales si se infunden al paciente ${ }^{(30)}$. Por lo tanto, debido a las limitaciones de compatibilidad y estabilidad de las mezclas de Nutrición Parenteral se requiere tener precaución en el agregado de hierro ${ }^{(21)}$ así como la influencia del Fe de contaminación en la estabilidad $y$ vida útil de las mezclas.

Harraki B, et al. han evidenciado que mezclas completas de NP se mostraron estables 7 días conservadas entre $4{ }^{\circ} \mathrm{C}$ y $25^{\circ} \mathrm{C}$ cuando la concentración de Fe era 


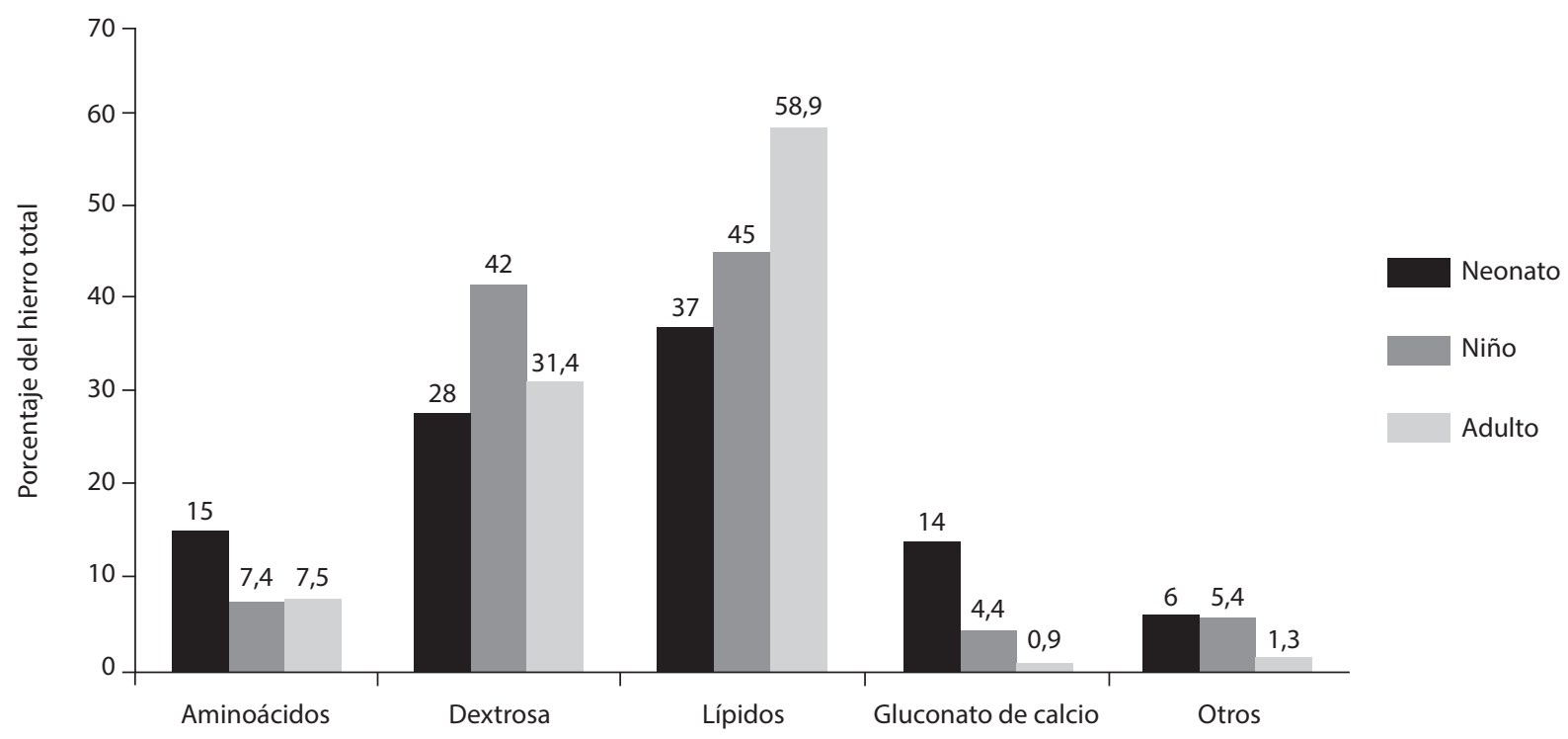

Figura 2. Porcentaje del hierro aportado por los componentes individuales en las mezclas de Nutrición Parenteral

inferior a $500 \mu \mathrm{g} / \mathrm{L}$. En el caso de superar esa cantidad comprobaron la desestabilización de los lípidos, por formación de grandes glóbulos de grasa, con importantes riesgos para los pacientes ${ }^{(37)}$.

En el presente trabajo la mezcla de NP para neonatología mostró un valor de $444 \mu \mathrm{g} / \mathrm{L}$, mientras que la de pediatría fue de $524 \mu \mathrm{g} / \mathrm{L}$ y la de adultos de $1176 \mu \mathrm{g} / \mathrm{L}$. Estas cifras sugieren la necesidad de realizar un control de estabilidad previo a la administración a los pacientes ya que la desestabilización se ve favorecida por altas temperaturas, luz, otro tipo de radiaciones; la presencia de metales como hierro, cobre, manganeso y de compuestos pro-oxidantes que aceleran la descomposición de hidroperóxidos.

En cambio, los complejantes de metales (aminoácidos u otros compuestos con capacidad quelante) y antioxidantes (vitamina $\mathrm{E}$ y otros) presentes en la mezcla retardan o inhiben la iniciación del proceso oxidativo.

\section{CONTENIDO DE HIERRO EN LOS PRODUCTOS FARMACÉUTICOS}

Las materias primas de los preparados farmacéuticos para administrar por vía inyectable deben cumplir con requisitos establecidos por las farmacopeas oficiales en cada país, en relación con los límites de impurezas presentes en los componentes que se utilizan para preparar NP. La Farmacopea argentina en su VII edición, establece el límite de hierro para pocos de los productos a administrar por vía inyectable, recomendando para su determinación un antiguo método colorimétrico de baja sensibilidad. Los métodos instrumentales (espectrometría de absorción atómica; IPCOES; Espectroscopía de Emisión Atómica - Plasma Inductivo acoplado a masa -IPC-MS) han representado un avance decisivo para la determinación de impurezas en los distintos productos farmacéuticos. Los trabajos publicados en las últimas décadas sobre la contaminación con elementos traza ${ }^{(6-10)}$ se han realizado mediante métodos instrumentales, como el utilizado en el presente trabajo, que es uno de los recomendados por la Farmacopea norteamericana (ICP-OES o ICP-MS), que aún no ha establecido un límite concreto para impurezas de $\mathrm{Fe}^{(38)}$ y la Europea que incluye cifras para los límites de exposición a impurezas por vía oral, parenteral e inhalación ${ }^{(11)}$.

Las Sociedades Científicas presentan controversia con respecto al agregado de $\mathrm{Fe}$ a la NP. En Estados Unidos los productos farmacéuticos multitraza no contienen $\mathrm{Fe}$, debido a la recomendación de no adicionarlo a la NP por su efecto sobre la estabilidad de los demás componentes de la fórmula. Sin embargo, en Europa la mayoría de los productos IV multitraza para neonatología, pediatría y adultos contienen $\mathrm{Fe}^{(21,39)}$. En algunos países de Suramérica (Argentina, Paraguay, Perú, etc.) existen productos multitraza para adultos que contienen Fe en su composición ( $2 \mathrm{mg}$ ). En este último caso, si el médico prescribe el agregado de soluciones multi- 
traza que contienen hierro, las cantidades presentes en la NP pueden superar los requerimientos del paciente $y$ ejercer efectos adversos sobre la evolución del paciente crítico, sobre todo en casos de NP prolongada, sepsis o en algunas patologías específicas (alteraciones hepáticas o renales) $)^{(40,41)}$.

\section{CONCLUSIONES}

Todos los componentes analizados, excepto el agua estéril, contenían Fe no declarado en los rótulos de los productos, con cantidades variables según el laboratorio y diferentes lotes del mismo fabricante.

Las mayores cantidades de Fe fueron encontradas en los lípidos, fosfato de sodio, gluconato de calcio y glicerofosfato de sodio. Los lípidos y la dextrosa contribuyeron con el mayor porcentaje de contaminación con Fe en las mezclas de NP.

La contaminación con Fe en las mezclas de NP de neonatos es muy baja con respecto a los requerimientos. En las fórmulas de niños el Fe de contaminación podría cubrir cerca de $70 \%$ de las necesidades y en el hombre adulto (no anémico) representaría el $200 \%$ de los requerimientos.

La concentración de Fe en las mezclas de NP pediátricas (neonatos y niños) se encontró cercana al límite de estabilidad $(500 \mu \mathrm{g} / \mathrm{L}$ de $\mathrm{Fe})$ y fue más del doble en la NP del adulto.

Los niveles de contaminación con Fe podrían aumentar la inestabilidad de los lípidos, así como las reacciones de oxidación de ciertos aminoácidos, vitaminas y ácidos grasos esenciales.

Cuando se agreguen productos multitraza para adultos que contienen $2 \mathrm{mg}$ de Fe a la mezcla de NP, la cantidad total de hierro aportada podría producir efectos adversos por sobredosis que pueden comprometer la evolución de los pacientes en estado crítico y de quienes reciben nutrición parenteral prolongada.

Es importante conocer el contenido de microminerales en los productos utilizados para preparar NP, para evitar las deficiencias y los excesos, que pueden comprometer la evolución del paciente y afectar la estabilidad de la mezcla de NP.

\section{Financiación}

Parcialmente financiado por la Universidad de Belgrano mediante subsidios para investigación.

\section{Conflicto de intereses}

Ninguno

\section{Referencias bibliográficas}

1. Boullata JI, Gilbert K, Sacks G et al. A.S.P.E.N. Board of Directors. Safe practices for parenteral Nutrition. Task Force for revision of Safe practices for parenteral Nutrition. JPEN J Parenter Enteral Nutr. 2014;28(Suppl):S39-S70.

2. Disposición Argentina, ANMAT- 2592/2003* Administración Nacional de Medicamentos, Alimentos y Tecnología Médica. Mezclas de Nutrición Parenteral Extemporáneas. Boletín Oficial de Argentina, Nro. 30162, junio-2003. Consultado mayo de 2018. Disponible en: http://www.anmat.gov.ar/webanmat/ Legislacion/Medicamentos/Disposicion_2592-03.pdf.

3. United States Pharmacopeia-USP 35. <797> Chapter. Pharmaceutical Compounding Sterile Preparations, Inc. Formulary 30. General Chapter Pharmaceutical Compounding-Sterile Preparations 2012:1-39. Consultado en mayo de 2018. Disponible en: https://www.snmmi.org/ files/docs/USP\%20797.pdf.

4. Portaría 272, Brasil. SIL. Ministério da Saúde. Secretaria de Vigilancia Sanitária. Regulamento técnico para a terapia de nutrição parenteral. Diario official da União República Federativa do Brasil, Brasilia Secção I, p.78,71-E, 15 de abril de 1998. Consultado en mayo de 2018. Disponible en: http:// www.anvisa.gov.br/anvisalegis/portarias/272_98.htm.

5. Marí AA, Jiménez Torres NV. Formulación de unidades nutrientes parenterales. Cap. 18. En: Mezclas Intravenosas y Nutrición Parenteral. Jiménez Torres V (eds.) Valencia, España: CONVASER. 1999. p. 469-501.

6. Fleming CR. Trace elements metabolism in adult patients requiring total parenteral nutrition. Am J Clin Nutr. 1989;49:573-9.

7. Hak EB, Storm MC, Helms RA. Chromium and zinc contamination of parenteral nutrition solution components commonly used in infants and children. Am J Health Syst Pharm. $1998 ; 15 ; 55(2): 150-4$.

8. Pluhator-Murton MM, Fedorak RN, Audette RJ, Marriage BJ, Yascoff RW and Gramlich R. Trace Element Contamination of TPN. 2. Effect of storage duration and temperature. JPEN. 1999;23:228-32.

9. Menéndez AM, Weisstaub AR, Montemerlo $H$, Rusi F, Guidoni M, Piñeiro A, Pita Martín de Portela ML. Contenido de zinc y cobre en los componentes individuales de las mezclas para fórmulas pediátricas de nutrición parenteral total. Nutrición Hospitalaria. 2007;22(5):545-51.

10. Menéndez AM, Farías, SS, Servant R, Morisio Y, Misischia Y, Simon S, Pita Martín de Portela ML. Contenido de aluminio en componentes individuales utilizados para preparar mezclas de nutrición parenteral en Argentina, y su comparación con 
la legislación internacional. Nutrición Hospitalaria. 2014; 29(6):1380-7.

11. Reddy MM, Reddy $\mathrm{KH}$ and Reddy MU. Harmonized Guideline on Limit and Testing of Elemental Impurities in Pharmaceutical Substances: A Review. Pharmaceut Reg Affairs. 2016,5(2):1-8. Disponible: https://www.omicsonline.org/open-access/harmonized-guideline-on-limit-andtesting-of-elemental-impurities-inpharmaceutical-substances-a-review-2167-7689-100016. Consultada: Julio /2018.

12. Farmacopea Argentina, Séptima Edición, Ministerio de Salud de la Nación Secretaría de Políticas, Regulación e Institutos ANMAT Administración Nacional de Medicamentos, Alimentos y Tecnología Médica INAME Instituto Nacional de Medicamentos. <580> Límite de hierro; 2013:287. Disponible: http://www.anmat.gov.ar/webanmat/fna/pfds/ Farmacopea_Argentina_2013_Ed.7.pdf.

13. Gordon DT, Peilett L. Physical and Chemistry properties of nutrients affecting their absorption and utilization. Chap 10. In: Physical Chemistry of Sahwartrberg HG and Hartel RW. Ed. Marcel Decker Inc. NY. Basel. H Kong. 1992.

14. Martín DW. Agua y Minerales. En: Bioquímica de Harper. Ed. El Manual Moderno S.A. de C.V., $10^{a}$ ed. México D.F. 1986.p.666-9.

15. Dallman PR. Iron. En: Present Knowledge in Nutrition. M.L.Brown Ed. Nutrition Foundation, Washington, D.C. $6^{\text {a }}$ ed. 1990. p. 241-50.

16. Gibson RS. Assessment of Iron Status. En: Principles of Nutritional Assessment. New York - Oxford. Oxford University Press. 1990. p. 349-76.

17. Yip R, Dallman PR. The roles of inflammation and iron deficiency as causes of anemia. Am. JClin. Nutr. 1988;48:1295-300.

18. Ovali F, Ciftci IH, Cetinkaya Z and Bukulmez A. Effects of a Human Milk- Fortifier on the Antimicrobial properties of Human Milk. J Perinatology. 2006;26:761-3.

19. Allwood MC and. Kearney MCJ. Compatibility and Stability of Additives in Parenteral Nutrition Admixtures. Nutrition. 1998;14:697-706.

20. Hardy G, Menéndez AM, Manzanares W. Suplementação de oligoelementos na Nutrição Parenteral. En: DL Waitzberg. Nutrição oral, Enteral e Parenteral na Prática Clínica. 4a. Edic. São Paulo: Atheneu. 2009. p.1043-54.

21. Vanek VW, et al. 2012. A.S.P.E.N. Position Paper: Recommendations for Changes in Commercially Available Parenteral Multivitamin and Multi-Trace Element Products. Nutrition in Clinical Practice. 2012; 27(4):440-91.

22. Food and Agricultural Organization/World Health Organization (FAO/WHO). Requirements of Vitamin A, Iron, Folate and Vitamin $\mathrm{B}_{12}$. Report of a Joint FAO/WHO Expert Group, WHO, Geneva, 1989.

23. Human Vitamin and Mineral Requirements. Report of a joint FAO/WHO expert consultation, WHO\&FAO, Ed. Roma. 2001. Consultado 30 de mayo de 2018. http://www.fao. org/3/a-y2809e.pdf.
24. Domellof M, Braegger C, Campoy C, Colomb V, Decsi T, Fewtrell $\mathrm{M}$, et al. Iron Requirements of Infants and Toddlers. On Behalf of the ESPGHAN Committee on Nutrition. JPGN. 2014;58:119-29.

25. Friel JK, Wayne LA, Hall MS, Rodway MS, Keith M, McCloy $\mathrm{CU}$, et al. Intravenous Iron Administration to Very-Low-BirthWeight Newborns Receiving Total and Partial Parenteral Nutrition. Journal of Parenteral and Enteral Nutrition. 1995;19(2):114-8. Disponible en: https://www.ncbi.nlm. nih.gov/pubmed/7609274. Consultado: agosto de 2018.

26. Comité Nacional de Hematología, Oncología y Medicina Transfusional, Comité Nacional de Nutrición. Deficiencia de hierro y anemia ferropénica. Guía para su prevención, diagnóstico y tratamiento. Arch Argent Pediatr. 2017;115 (Supl 4):s68-s82.

27. Cerami C. Iron Nutriture of the Fetus, Neonate, Infant and Child. Ann Nutr Metab. 2017;71(suppl 3):8-14.

28. Adamkin DH. Feeding in preterm infant. In: Perinatal Nutrition Optimizing Infant Health and Developmental. J Bathis, Ed. New York: Marcel Dekker, 2004;165-90.

29. Boveris A. Biochemistry of free radicals: from electrons to tissues. Medicina. Buenos Aires, Argentina. 1998;58:350-6.

30. Steger PJK, Mühlebach SF. Lipid Peroxidation of Intravenous Lipid Emulsions and All-in-One Admixtures in Total Parenteral Nutrition Bags: The Influence of Trace Elements. JPEN J Parenter Enteral Nutr. 2000;24:37-41.

31. Bacon BR, Tavill AS, Brittenham GM, Park CH and Recknagel RO. Hepatic lipid peroxidation in vivo in rats with chronic iron overload, J.Clin Invest. 1983;71:429-39.

32. Salonen JT, Nyyssonen K, Korpela H, Tuomilehto J, Seppanen R, Salonen R. High stored iron levels are associated with excess risk of myocardial infarction in Eastern Finnish men. Circulation. 1992;86:803-11.

33. International Committee for the Classification of Retinopathy of Prematurity: The International Classification of Retinopathy of Prematurity revisited. Arch Ophthalmol. 2005;7:991-9.

34. Lomuto CC. Grupo de Trabajo colaborativo multicéntrico: Prevención de la ceguera en la infancia por Retinopatía del Prematuro (ROP). En: Prevención de la ceguera en la infancia por ROP. Cap 7. Buenos Aires, Ministerio de Salud-UNICEF. 2008 (15):49-53.

35. Hauer EC, Kaminski MV. Trace metal profile of parenteral nutrition solutions. Am J Clin Nutr. 1978;31:264-8.

36. Halliwell B. Antioxidants and human disease: a general introduction. Nutr Rev. 1997;55(1):S44-S52.

37. Harraki B, Guiraud P, Rochat MH, et al. Influence of copper, iron, and zinc on the physicochemical properties of parenteral admixture. J Parenter Sci Technol. 1993;47(5):199-204.

38. United States Pharmacopeia-USP. Parenteral Limit. Elemental Impurities: Standards-Setting Record. December 20, 2012:86.

39. Consultado en julio de 2018. Disponible en: http://www. usppf.com/pf/pub/data/v345/GEN_STIMULI_345_ pf345-stim1.xml[12/7/2012 1:29:00 PM]. 
40. Shenkin A. Trace elements and vitamins in parenteral and enteral nutrition. In: Basics in Cinical Nutrition. L Sobotka, et al. Edited for ESPEN. Fourth Ed. Prague, Czech Republic: Hause Galen. 2011. p. 273-7.

41. Tenenbein M. Toxicokinetics and Toxicodynamics of iron poisoning. Toxicology Letters. 1998;(102-103):653-6.
42. Yukata Kohgo, Katsuya Ikuta, Takaaki Ohtake, Yoshihiro Torimoto and Junji Kato. Body iron metabolism and pathophysiology of iron overload. Int J Hematol. 2008;88:7-15. 